\title{
Structure and Assembly of the Bacillus anthracis Exosporium
}

\author{
Cynthia M Rodenburg, Sylvia A McPherson, Charles L. Turnbough Jr., Terje Dokland \\ Department of Microbiology, University of Alabama at Birmingham, Birmingham, AL, USA
}

Bacillus anthracis, the causative agent of anthrax, is a highly pathogenic Gram-positive soil bacterium that is also considered a serious threat as a potential bioterrorism agent. Like other bacilli, B. anthracis forms protective spores upon starvation. The spores are the main pathogenic agents, easily spread and highly resistant to environmental stress. When the spores enter a mammalian host, they germinate and undergo vegetative growth, followed by production of anthrax toxin, which leads to systemic bacteremia and toxemia that is often fatal.

The spore is a complex, multilayered structure, consisting of a nucleoid surrounded by a peptidoglycan cortex and a proteinaceous coat [1]. Spores of B. anthracis and other pathogenic bacilli are surrounded by an exosporium, a balloon-like layer that acts as the outer permeability barrier of the spore and is thought to contribute to spore survival in the environment, uptake by potential hosts, and as a virulence determinant. The exosporium consists of a hair-like nap and a paracrystalline basal layer [1]. The filaments of the nap are comprised of trimers of the collagen-like glycoprotein BclA, while the basal layer contains at least 20 different proteins. One of these proteins, BxpB, forms tight complexes with BclA and is required for attachment of essentially all BclA filaments to the basal layer [2]. Another basal layer protein, ExsB, is required for the stable attachment of the exosporium to the spore [3]. ExsY is required to complete exosporium assembly during sporulation [4]. The exosporium also harbors several enzymes, including alanine racemase (Alr), which functions as an anti-germinant, presumably functioning to prevent premature germination.

We have used a combination of cryo-electron microscopy (cryo-EM), cryo-sectioning (Tokuyasu method) and crystallographic analysis of negatively stained exosporium fragments to compare wildtype spores and spores with deletions of specific exosporium genes, including $b c l A, \operatorname{bxp} B$, exs $B$ and exs $Y$, to study the structure and assembly of the B. anthracis exosporium [5].

Cryo-EM reveals the intact spore in its native form and shows clearly the bilayered basal layer and the nap filaments with their distal knobs (Fig. 1A, B). The filaments appear to connect to protrusions in the basal layer, more clearly seen in negatively stained cryo-sections (Fig. 1C). $\Delta b c l A$ spores lack the nap, while $\triangle b x p B$ spores lack both the nap and the protrusions, but retain the bilayered basal layer (Fig. 1D, $\mathrm{E})$. The exosporium of $\Delta e x s B$ spores is more fragile and is easily sloughed upon grown in liquid media, but looks otherwise completely normal and contains a normal complement of BxpB protrusions and BclA filaments (Fig. 1F). Taken together with crystallographic analysis of isolated exosporium fragments, these data suggest that the protrusions are made of trimers of BxpB (Fig. 1G, H). The protrusions interact with a crystalline layer of hexagonal subunits formed by other, still undetermined basal layer proteins (Fig. 1G). Although $\triangle b x p B$ spores retain the hexagonal subunits, the basal layer is not organized with crystalline order and lacks basal layer protrusions and most BclA filaments, indicating a central role for BxpB in exosporium organization. 


\section{References:}

[1] Henriques, A.O. and Moran Jr., C.P., (2007). Annu. Rev. Microbiol. 61, 555-588.

[2] Steichen, C. T., Kearney, J. F., and Turnbough, C. L., Jr. (2005). J. Bacteriol. 187, 2868-5876

[3] McPherson, S., Li, M., Kearney, J. and Turnbough Jr., C.L. (2010). Mol. Microbiol. 76, 1527-1538.

[4] Boydston, J.A., Yue, L., Kearney, J.F., and Turnbough, Jr., C.L. (2006) J. Bacteriol. 188, 7440-7448

[5] Rodenburg, C.M., McPherson, S.A., Turnbough Jr., C.L. and Dokland, T. (2014). J. Struct. Biol. 186, $181-187$.
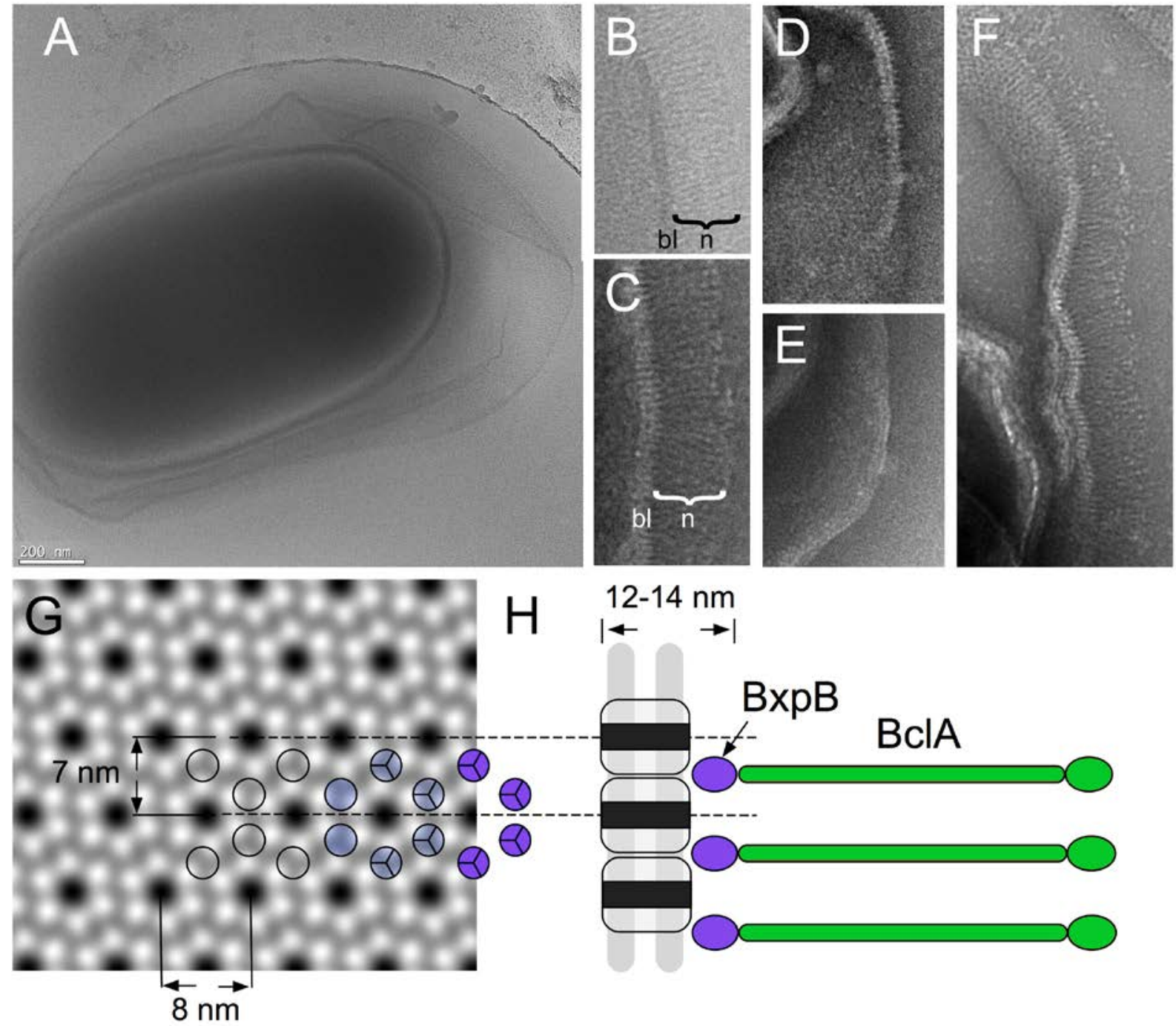

Figure 1. (A) Cryo-electron micrograph of the wildtype B. anthracis spore. (B) Cryo-EM (high-pass filtered) and (C) negatively stained cryo-section of the wildtype exosporium, showing the nap (n) and basal layer (bl). (D-F) Cryo-sections of the $\Delta b c l A(\mathrm{D}), \Delta b x p B$ (E) and $\Delta \operatorname{exs} B$ (F) exosporium. (G) 2D crystal average of the negatively stained, wildtype exosporium, showing the hexagonal subunits. BxpB trimers are indicated in purple. (H) Model for the arrangement of BxpB (purple) and BclA (green). 